Proceedings of the Edinburgh Mathematical Society (2005) 48, 631-634 (C)

DOI:10.1017/S0013091504000604 Printed in the United Kingdom

\title{
THE LINEAR SPAN OF PEAK FUNCTIONS
}

\author{
ALEXANDER J. IZZO \\ Department of Mathematics and Statistics, Bowling Green State University, \\ Bowling Green, OH 43403, USA (aizzo@math.bgsu.edu)
}

(Received 7 July 2004)

\begin{abstract}
It is shown that given a uniform algebra $A$ and a peak point $x_{0}$ for $A$, every function in $A$ can be expressed as a linear combination of two functions in $A$ that peak at $x_{0}$.

Keywords: uniform algebra; peak point; linear combination

2000 Mathematics subject classification: Primary 46J10; 46J15; 30H05
\end{abstract}

In their work on contractive actions on a kernel Hilbert space [1], Davidson and Douglas were led to the following question: is the linear span of the functions in the ball algebra $A\left(B_{2}\right)$ that peak at the point $(1,0)$ uniformly dense in the ball algebra $A\left(B_{2}\right)$ ? (The ball algebra $A\left(B_{2}\right)$ consists of the continuous functions on the closed unit ball $\bar{B}_{2}$ in $\mathbb{C}^{2}$ that are holomorphic on the open unit ball $B_{2}$. A function $f$ is said to peak at a point $x_{0}$ if $f\left(x_{0}\right)=1$ and $|f(x)|<1$ for all other points $x$ in the domain of $f$.) It turns out that the answer is affirmative. Moreover, the linear span is not only dense in the ball algebra, it is equal to the ball algebra, and in fact every function in the ball algebra can be expressed as a linear combination of two functions in the ball algebra that peak at $(1,0)$. Furthermore, this fact carries over to arbitrary uniform algebras. After this information was communicated to Douglas and Davidson, they found an entirely different proof of the density of the linear span of peak functions in the ball algebra. See [1] for their proof and applications.

Here now is the precise statement of our result. (A point $x_{0}$ is a peak point for a uniform algebra if there is a function in the algebra that peaks at $x_{0}$.)

Theorem 1. Let $A$ be a uniform algebra on a compact Hausdorff space $X$, and let $x_{0}$ be a peak point for $A$. Then every function in $A$ can be expressed as a linear combination of two functions in $A$ that peak at $x_{0}$.

We will establish this theorem using the following result.

Proposition 2. Let $A$ be a uniform algebra on a compact Hausdorff space $X$, and let $x_{0}$ be a peak point for $A$. Let $p$ be a positive continuous function on $X$. Then there is a function $g$ in $A$ such that $g\left(x_{0}\right)=p\left(x_{0}\right)$ and $|g(x)| \leqslant p(x)$ for all $x \in X$. 
This proposition is a special case of the following standard fact from uniform algebra theory, which appears as Theorem II.12.5 in [2].

Theorem 3. Let $B$ be a closed subspace of $C(X)$ ( $X$ a compact Hausdorff space). Let $E$ be a closed subset of $X$ such that, for every measure $\mu$ that annihilates $B$, the restriction $\mu_{E}$ of $\mu$ to $E$ also annihilates $B$. Let $\left.f \in B\right|_{E}$, and let $p$ be a positive continuous function on $X$ such that $|f(y)| \leqslant p(y)$ for all $y \in E$. Then there is $g \in B$ such that $\left.g\right|_{E}=f$ and $|g(x)| \leqslant p(x)$ for all $x \in X$.

To see that Proposition 2 is a special case of Theorem 3 note that if $B$ is a uniform algebra, $x_{0}$ is a peak point for $B$, the function $h \in B$ peaks at $x_{0}$, and $\mu$ annihilates $B$, then

$$
\mu\left(\left\{x_{0}\right\}\right)=\lim _{n \rightarrow \infty} \int h^{n} \mathrm{~d} \mu=0,
$$

so the singleton set $\left\{x_{0}\right\}$ satisfies the hypothesis on the set $E$ above. On the other hand, the use of Theorem 3 can be avoided, for, as pointed out by the referee, there is a completely elementary proof of Proposition 2 which we now present.

Proof of Proposition 2. Let $h \in A$ peak at $x_{0}$. We can suppose that $p\left(x_{0}\right)=h\left(x_{0}\right)=$ 1 and that for all $x \in X$ we have $p(x) \geqslant \delta>0$ and $|h(x)|<\frac{3}{2} p(x)$. For each positive integer $n$, set

$$
S_{n}=\left\{x \in X: \frac{|h(x)|}{p(x)} \in\left(1+\frac{1}{2^{n+1}}, 1+\frac{1}{2^{n}}\right]\right\} .
$$

Note that each $S_{n}$ is disjoint from a neighbourhood of $x_{0}$, and hence for each $n$ there is an $\eta_{n}$ such that $|h(x)|<\eta_{n}<1$ for all $x \in S_{n}$. Hence for each $n$ we can choose a positive integer $k_{n}$ such that $|h(x)|^{k_{n}}<\delta / 3^{n}$ for all $x \in S_{n}$. Now define

$$
g=\sum_{n=1}^{\infty} \frac{h^{k_{n}}}{2^{n}}
$$

so that $g$ is in $A$. Note that for every $m$ and $n$ and $x \in S_{m}$ we have

$$
|h(x)|^{k_{n}} \leqslant|h(x)| \leqslant\left(1+\frac{1}{2^{m}}\right) p(x)
$$

(as $|h(x)| \leqslant 1$ ). Thus, for $x \in S_{m}$, we have

$$
|g(x)| \leqslant \sum_{n}\left\{\left(\frac{1}{2^{n}}\right)\left(1+\frac{1}{2^{m}}\right) p(x): n \neq m\right\}+\left(\frac{1}{2^{m}}\right)\left(\frac{1}{3^{m}}\right) p(x)<p(x) .
$$

Also, for $x \in X \backslash \cup S_{n}$, it is clear that $|g(x)| \leqslant p(x)$. Finally, $g\left(x_{0}\right)=1$.

Proof of Theorem 1. Let $f$ be a function in $A$. Consider first the case when $f\left(x_{0}\right)=0$. Choose a continuous function $\varphi$ that peaks at $x_{0}$, and set $\alpha(x)=1-|\varphi(x)|$. Then $\alpha$ is a non-negative continuous function on $X$ that vanishes only at $x_{0}$. Now choose $M>\|\alpha\|_{\infty}+\|f\|_{\infty}$ and set

$$
p(x)=M-\alpha(x)-|f(x)| .
$$

Then $p$ is a positive continuous function on $X$, and $p$ has a strict maximum at $x_{0}$. 
By Proposition 2, there is a function $g$ in $A$ such that $g\left(x_{0}\right)=p\left(x_{0}\right)$ and $|g(x)| \leqslant p(x)$ for all $x \in X$. Then $|g(x)|<g\left(x_{0}\right)$ for all $x \neq x_{0}$, so some scalar multiple of $g$ peaks at $x_{0}$.

Now set $h=g+f$. Then $h \in A$ and

$$
h\left(x_{0}\right)=g\left(x_{0}\right)+f\left(x_{0}\right)=g\left(x_{0}\right)=M .
$$

For all $x \in X$,

$$
\begin{aligned}
|h(x)| & \leqslant|g(x)|+|f(x)| \\
& \leqslant p(x)+|f(x)| \\
& =M-\alpha(x) .
\end{aligned}
$$

Thus $|h(x)|<h\left(x_{0}\right)$ for all $x \neq x_{0}$, so some scalar multiple of $h$ peaks at $x_{0}$. Since $f=h-g$, this completes the proof in the case when $f\left(x_{0}\right)=0$.

For the general case, note that we may assume without loss of generality that $f\left(x_{0}\right) \geqslant 0$.

From the case already considered we have

$$
f-f\left(x_{0}\right)=h-g
$$

with $g$ and $h$ scalar multiples of functions that peak at $x_{0}$ and with $h\left(x_{0}\right)=g\left(x_{0}\right)=$ $M>0$. Now $h+f\left(x_{0}\right)$ takes a strict maximum at $x_{0}$ and hence is a scalar multiple of a function that peaks at $x_{0}$. Since $f=\left(h+f\left(x_{0}\right)\right)-g$, this completes the proof.

Remark 4. A slight modification of the above argument shows that when $f\left(x_{0}\right)=0$ and $\|f\|_{\infty}<1$, then $f=h-g$, where each of $g$ and $h$ peaks at $x_{0}$. We simply replace $\alpha$ by $\epsilon \alpha$, where $\epsilon>0$ is a number small enough that $\|\epsilon \alpha\|_{\infty}+\|f\|_{\infty}<1$, and set $M=1$. The last part of the above argument can then be arranged to show that more generally whenever $f\left(x_{0}\right)$ is real, $f=h-g$ with $g$ and $h$ positive scalar multiples of peak functions.

Remark 5. If the space $X$ is not metrizable, then the algebra $A$ may not have any peak points, but it will still have $p$-points (see $[2, \S$ II.12]). A version of Theorem 1 remains true for $p$-points. Omitting $\varphi$ and $\alpha$ from the proof of Theorem 1, we see that every function in $A$ is a difference of two functions $g$ and $h$ in $A$ each attaining its maximum modulus at $x_{0}$, but these maxima may not be strict. Taking $\varphi$ to be a function that peaks only 'near' $x_{0}$, one can arrange to have the maxima of $g$ and $h$ attained only in an arbitrarily (small) prescribed neighbourhood of $x_{0}$.

Remark 6. It is well known that Theorem 3 (together with the F. and M. Riesz theorem) yields the Rudin-Carleson theorem that every closed subset $E$ of the unit circle with linear measure zero is a peak-interpolation set for the disc algebra. It is interesting to note that Theorem 3 also implies that the interpolating function can be required to decrease arbitrarily fast off of $E$. In particular, it shows that there are functions in the disc algebra that peak arbitrarily steeply at the point 1 .

Acknowledgements. This paper grew out of discussions I had with John Wermer, for which I thank him. I also thank the Department of Mathematics at Brown University, where this work was carried out, for its hospitality during my time there. In addition, I thank the referee for providing the elementary proof of Proposition 2 given above. 


\section{References}

1. K. R. Davidson and R. G. Douglas, The generalized Berezin transform and commutator ideals, Pac. J. Math., in press.

2. T. W. Gamelin, Uniform algebras, 2nd edn (Chelsea, New York, 1984). 